\title{
Influencia de la Paridad en el Peso del Recién Nacido en Hospitales del Ministerio de Salud del Perú
}

\section{Influence of the Parity on the Weight of the Newborn in Hospitals of the Health Ministry of the Peru}

\author{
${ }^{1}$ Manuel Ticona Rendón, ${ }^{2}$ Diana Huanco Apaza, ${ }^{3}$ Maricarmen Ticona Vildoso
}

\begin{abstract}
RESUMEN:
El objetivo del estudio fue determinar influencia de paridad en peso de neonato en Hospitales del Ministerio de Salud. La metodología se realizó mediante un Estudio Transversal y analítico en $485+5$ neonatos vivos de 37 a $\$ 2$ semanas de gestación sin factores de desnutrición intrauterina, de madres sanas, nacidos en 29 hospitales del Ministerio de Salud del Perú en 2005. Se usó base de datos del Sistema Informático Perinatal. Estadísticos usados: promedio, desviación estándar, percentil 50, chi cuadrado y t de Student. Los resultados obtenidos fueron que el promedio de peso de neonatos fue $3306 \mathrm{~g}$. $+432 \mathrm{~g} ., 58.2 \%$ provienen de primiparas, con asociación directa entre paridady peso de neonatos $(p<0.01)$. Hijos de primíparas tienen $26 \mathrm{~g}$. de peso menos que población general, multiparas y gran multiparas 35 y $58 \mathrm{~g}$. mayor respectivamente $(p<0.01$ ). gran multiparas presentaron mayor proporción de bajo y alto peso y primiparas peso insuficiente. En conclusión se pudo determinar que la Paridad influye significativamente en peso de neonato a término de hospitales del Ministerio de Salud del Perí.
\end{abstract}

Palabras clave: paridad, peso del recién nacido

\section{ABSTRACT:}

The objective of study was to determine the parity influence on the weight of newborn in Hospitals of Health Minister: The methodology was performed using a transversal and analytical study in 48545 alive newborns with 37 to 42 gestation weeks without factors of intrauterine malnutrition, healthymothers's, born in 29 hospitals of Health Minister in Peru in 2005. Data base of the System Informatics Perinatal was used. Statistics used: Average, standard deviation, percentile 50, chi square and t-student. Obtained the results were $3306 \mathrm{~g}+432$ gestates was newborn weighty average, $58.2 \%$ primiparity, Children have $26 \mathrm{~g}$. weighty less than general population, multiparity and great multiparity 35 and 58 bigger g. respectively $(p<0.01)$, number of multiparity showed higher proportion of low and high weight and primiparity insufficient weigh. In conclusion it could be determined that Parity influences significantly on the newhorn weight in hospitals of Health Minister in Peru.

Keywords: parity, iweigh of the newborn

\footnotetext{
' Médico Pediatra y Neonatólogo. Doctor en Salud Pública. Facultad de Ciencias de la Salud. Universidad Nacional Jorge Basadre Grohmann

${ }^{2}$ Doctora en Salud Pública. Licenciada en Obstetricia. Hospital Hipólito Unanue de Tacna.

'Médico Residente de Pediatría. Hospital Honorio Delgado de Arequipa.
} 


\section{INTRODUCCIÓN}

El crecimiento intrauterino valorado por peso, talla y perimetro craneal, constituyen en conjunto medidas antropométricas y al misıno tiempo son buenos indicadores de salud y de madurez fetal.

El crecimiento intrauterino implica un proceso de sintesis de moléculas simples a bio moléculas complejas, que se produce simultáneamente con la diferenciación celular y que lleva a la formación de órganos y tejidos con funciones complejas e interrelacionadas. Aunque los procesos íntimos son desconocidos, puede ser modificado por numerosos factores. El $60 \%$ de la variación del peso al nacer puede ser atribuida al entorno en que el feto crece (1).

El crecimiento intrauterino puede estar influenciado por: estado nutricional, antecedentes obstétricos, peso, talla materna, hábitos (tabaco, alcohol, drogas), peso de la placenta, sexo fetal, intervalo intergenésico, orden de nacimiento y edad materna.

La paridad como factor preconcepcional es importante, se ha comprobado que el peso del primer hijo es menor que el de los subsiguientes: asi como también, las curvas de crecimiento intrauterino para primogénitos, muestran en las 38 semanas de amenorrea, un peso promedio $100 \mathrm{~g}$. menor que las curvas de neonatos hijos de madres secundigestas. Niswander y Gordon observaron que el peso promedio de los productos va aumentando, desde el segundo hijo hasta el quinto, descendiendo a partir del sexto (1).

El efecto de la paridad por sí sola, sobre el peso de los neonatos es muy discutido. Es probable que el descenso del peso promedio de los RN a partir del quinto hijo, se debería más a condiciones socio económicas desfavorables, que al factor paridad. Por otra parte, se sabe que las primigestas presentan más frecuentemente toxemia, enfermedad que determina mayor incidencia de neonatos de bajo peso; a la inversa, la diabetes que se relaciona con fetos de peso elevado, predomina en las multiparas.

El objetivo de este estudio fue determinar la influencia de la paridad en el comportamiento del crecimiento fetal en las edades gestacionales de 37 a 42 semanas en hospitales del Ministerio de Salud del Perú.

\section{MATERIAL Y MÉTODOS}

Estudio transversal y analitico en 48545 recién nacidos vivos de 37 a 42 semanas de gestación, sin factores de retardo de crecimiento intrauterino, de madres sanas, nacidos en 29 hospitales del Ministerio de Salud del Perú durante el año 2005.

La paridad fue consignada en número de partos ocurridos inclusive el actual, esta información se obtuvo por interrogatorio directo a la madre y el mismo que se encuentra registrado en la historia clínica perinatal.

Los datos se analizan en forma independiente por cada paridad hasta 7 a más, para la clasificación y estudio del número total de gestaciones se confeccionó una escala que los ubicó en tres grupos determinados de la siguiente forma:

Grupo I:

Grupo II:

Grupo III:

Los hijos de primíparas.

Los hijos de multíparas (2 a 4 partos).

Los productos de gran multiparas ( 5 a más partos).

El peso del RN se clasificó de acuerdo con las siguientes categorias:

- Bajo peso (menos de $2500 \mathrm{~g}$.)

- Peso insuficiente (2500 a menos de $3000 \mathrm{~g}$.)

- Peso adecuado (3000 a 3999 g.)

- Peso alto (4000 g. a más)

Los estadisticos usados fueron: promedios, desviación estándar, percentil 50 . chi cuadrado y prueba $t$ de Student. con una confiabilidad del $95 \%$, considerando significativo cuando $p<0.05$.

El procesamiento de datos y el análisis estadístico se realizó con el Sistema Informático Perinatal (SIP2000) y el programa Epi-Info.

\section{RESULTADOS}

De los 48545 recién nacidos estudiados, $58.16 \%$ provienen de madres primiparas, disminuyendo este porcentaje conforme aumenta la paridad, llegando a $1.19 \%$ en las madres con paridad de 7 a más (Tabla y Figura 1).

Tabla 1. Distribución de Paridad

\begin{tabular}{lcc}
\hline Paridad & No. & \% \\
\hline 1 & 28235 & 58.2 \\
2 & 10172 & 21.0 \\
3 & 5336 & 11.0 \\
4 & 2428 & 5.0 \\
5 & 1192 & 2.5 \\
6 & 603 & 1.2 \\
7 a más & 579 & 1.2 \\
Total & $\mathbf{4 8 5 4 5}$ & $\mathbf{1 0 0 , 0}$ \\
\hline
\end{tabular}

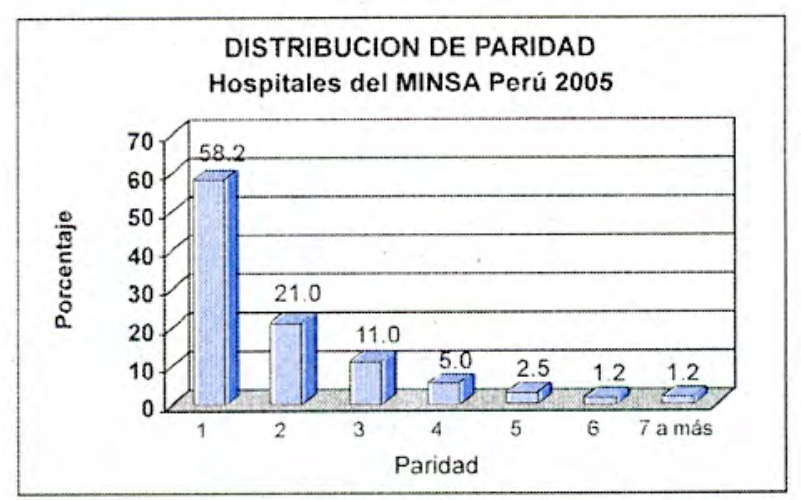

Figura 1. Distribución de Paridad de los Hospitales del MINSA Perú 2005

Fuente: Sistema Informático Perinatal de Hospitales del MINSAPerú. 
El peso promedio de los recién nacidos se incrementó conforme aumenta la paridad, hasta la paridad 5 y luego disminuyó; asi. las madres primiparas, presentaron un promedio de peso de sus recién nacidos de $3280 \mathrm{~g}$. y las madres con paridad cinco, $3380 \mathrm{~g}$. (Tabla 2 y Figura 2).

Tabla 2. Paridad y Peso Promedio del RN

\begin{tabular}{ccc}
\hline Paridad & Peso RN & DS \\
\hline 1 & 3280 & 423 \\
2 & 3333 & 421 \\
3 & 3351 & 450 \\
4 & 3355 & 457 \\
5 & 3380 & 476 \\
6 & 3376 & 464 \\
7 a más & 3317 & 527 \\
Población Total & 3306 & $\mathbf{4 3 2}$ \\
\hline
\end{tabular}

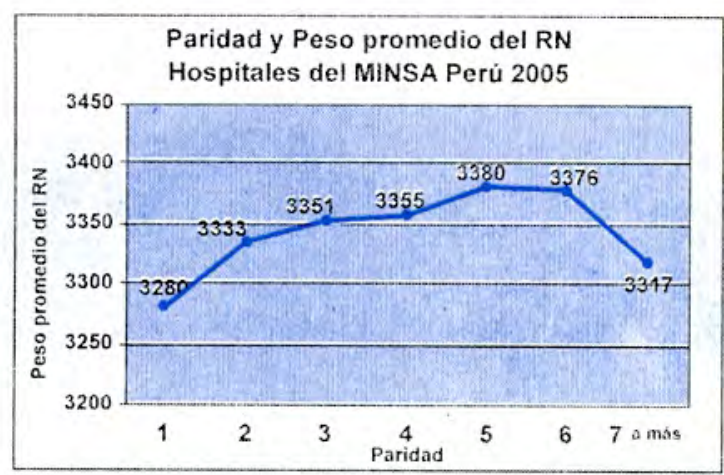

Figura 2. Paridad y Peso promedio del $R N$ en Hospitales del MINSA Perú 2005

Fuente: Sistema Informático Perinatal de Hospilales del MINSAPerú.

Los recién nacidos de madres primiparas presentaron 26 g. menos que la población general, las multiparas y las grandes multiparas 35 y $58 \mathrm{~g}$. más respectivamente, siendo estas diferencias estadisticamente significativas $(p<0.01)$. La diferencia del peso al nacer entre las primíparas y las de paridad 5 es de $100 \mathrm{~g}$. (Tabla $3 \mathrm{y}$ Figura 3).

Tabla 3. Paridad y Peso promedio del RN

\begin{tabular}{lcccc}
\hline \multicolumn{1}{c}{ Paridad } & No. & Peso RN & DS & P \\
\hline Primípara (1) & 28235 & 3380 & 476 & 0.010 \\
Multípara (2-4) & 17936 & 3376 & 464 & 0.003 \\
$\begin{array}{l}\text { Gran multipara } \\
\text { (5 a +) }\end{array}$ & 2374 & 3317 & 527 & 0.000 \\
$\begin{array}{l}\text { Población } \\
\text { Total }\end{array}$ & $\mathbf{4 8 5 4 5}$ & $\mathbf{3 3 0 6}$ & $\mathbf{4 3 2}$ & \\
\hline
\end{tabular}

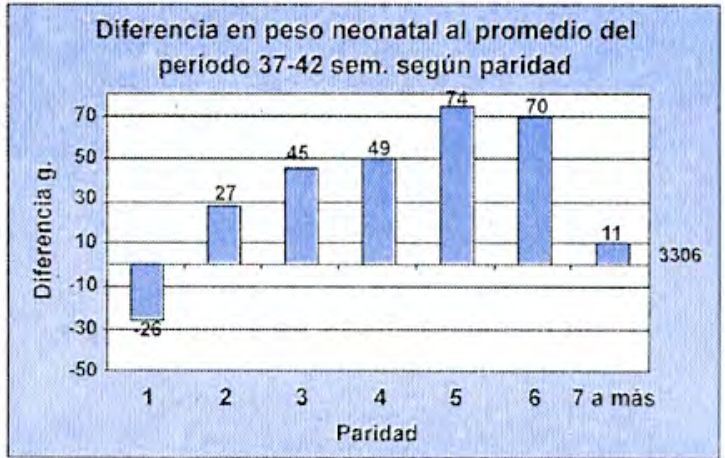

Figura 3. Diferencia en peso neonatal al promedio del periodo $37-42 \mathrm{sem}$. según paridad

Fuente: Sistema Informático Perinatal de Hospitales del MINSA-Perú.

El percentil 50 de peso del recién nacido en las edades gestacionales de 37 a 42 semanas en madres primíparas están por debajo de la población general. siendo estas diferencias estadisticamente significativas ( $p<0.01) ; \sin$ embargo los pesos de madres multiparas y gran multiparas es superior a la población general, pero las diferencias no son significativas. (Tabla 4 y Figura 4).

Tabla 4. Peso del RN (percentil 50) de acuerdo a grupos de paridad

\begin{tabular}{lcccccc}
\hline Paridad & \multicolumn{5}{c}{ Edad gestacional en semanas } \\
& $\mathbf{3 7}$ & $\mathbf{3 8}$ & $\mathbf{3 9}$ & $\mathbf{4 0}$ & $\mathbf{4 1}$ & $\mathbf{4 2}$ \\
\hline $\mathrm{l}$ & 2976 & 3149 & 3254 & 3364 & 3469 & 3503 \\
$2-4$ & 3056 & 3239 & 3344 & 3445 & 3519 & 3504 \\
5 a + & 2980 & 3242 & 3379 & 3483 & 3499 & 3470 \\
Pob. Total & $\mathbf{3 0 1 2}$ & $\mathbf{3 1 9 1}$ & $\mathbf{3 2 9 5}$ & $\mathbf{3 3 9 7}$ & $\mathbf{3 4 8 7}$ & $\mathbf{3 4 9 8}$ \\
\hline
\end{tabular}

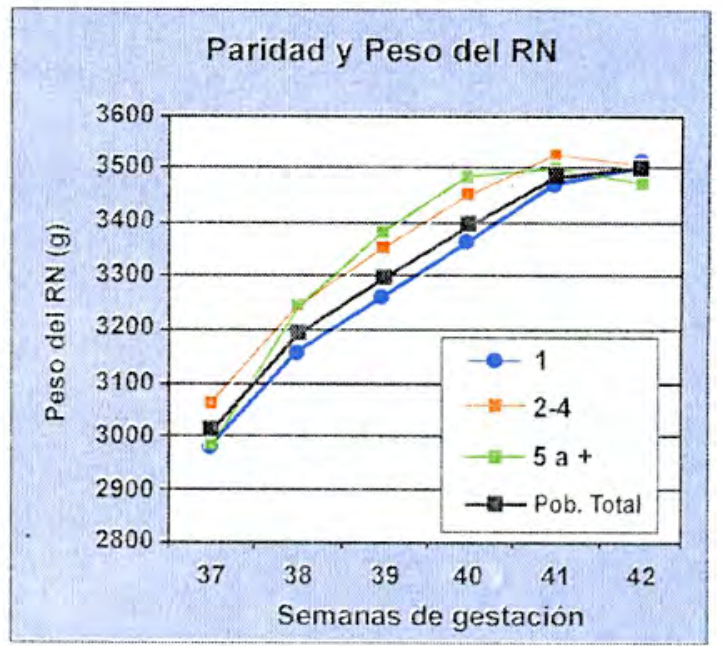

Figura 4. Paridad y Peso del RN

Fuente: Sistema Intormático Perinatal de Hospitales del MINSAPeru.

Tabla 5. Paridad y Categorias de Peso del Recién Nacido

\begin{tabular}{lcccccccccc} 
& \multicolumn{3}{c}{ Escolaridad } & \multicolumn{2}{c}{ Bajo peso } & \multicolumn{2}{c}{ Peso } & \multicolumn{2}{c}{ Insuficiente } & \multicolumn{2}{c}{ Peso adecuado } & \multicolumn{2}{c}{ Peso alto } & \multicolumn{2}{c}{ TOTAL } \\
& No. & \% & No. & \% & No. & \% & No. & $\%$ & No. & $\%$ \\
\cline { 2 - 12 } Primípara & 691 & 2.4 & 5932 & 21.0 & 20200 & 71.5 & 1412 & 5.0 & 28235 & 100.0 \\
Multipara & 368 & 2.1 & 3165 & 17.6 & 13168 & 73.4 & 1235 & 6.9 & 17936 & 100.0 \\
Gran multíp. & 83 & 3.5 & 377 & 15.9 & 1687 & 71.1 & 227 & 9.6 & 2374 & 100.0 \\
Pob. general & $\mathbf{1 1 4 2}$ & $\mathbf{2 . 4}$ & $\mathbf{9 4 7 4}$ & $\mathbf{1 9 . 5}$ & $\mathbf{3 5 0 5 5}$ & $\mathbf{7 2 . 2}$ & $\mathbf{2 8 7 4}$ & $\mathbf{5 . 9}$ & $\mathbf{4 8 5 4 5}$ & $\mathbf{1 0 0 . 0}$ \\
\hline
\end{tabular}


Las madres gran multiparas tienen mayor porcentaje de recién nacidos con bajo peso, mientras que las primiparas tienen mayor porcentaje de recién nacidos con peso insuficiente. Existe un incremento de la frecuencia de alto peso conforme aumenta la paridad, así las gran multíparas presentaron dos veces mayor porcentaje de alto peso respecto a las primíparas, existiendo asociación directa entre la paridad y las categorías de peso del recién nacido $(\mathrm{p}<0.01)$ (Tabla 5 , Figuras 5,6 y 7).

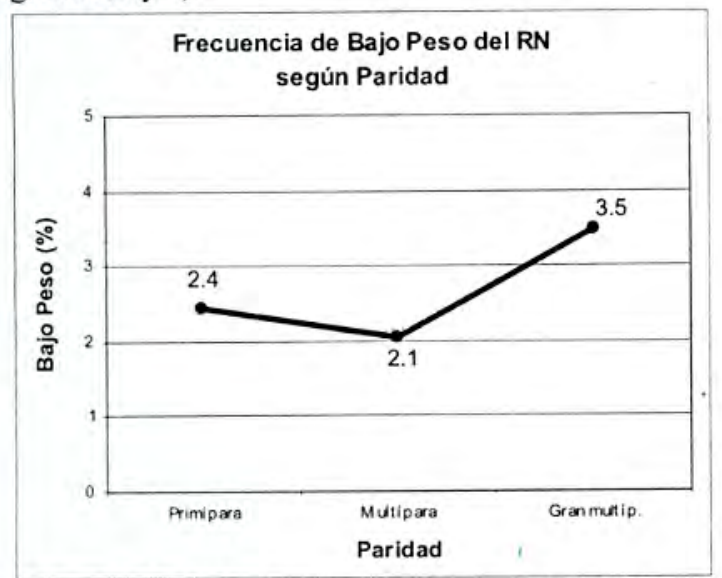

Figura 5. Frecuencia de Bajo Peso del RN según paridad

Fuente: Sistema Informático Perinatal de Hospitales del MINSAPerú.

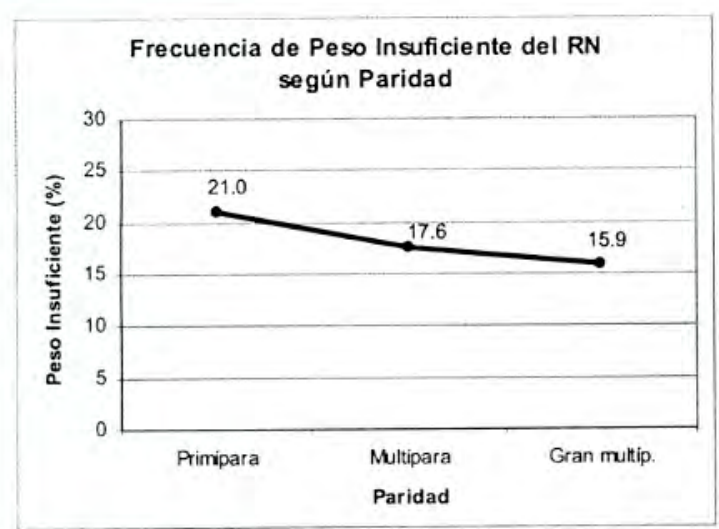

Figura 6. Frecuencia de Peso Insuficiente del RN según paridad

Fuente: Sistema Informático Perinatal de Hospitales del MINSAPerú.

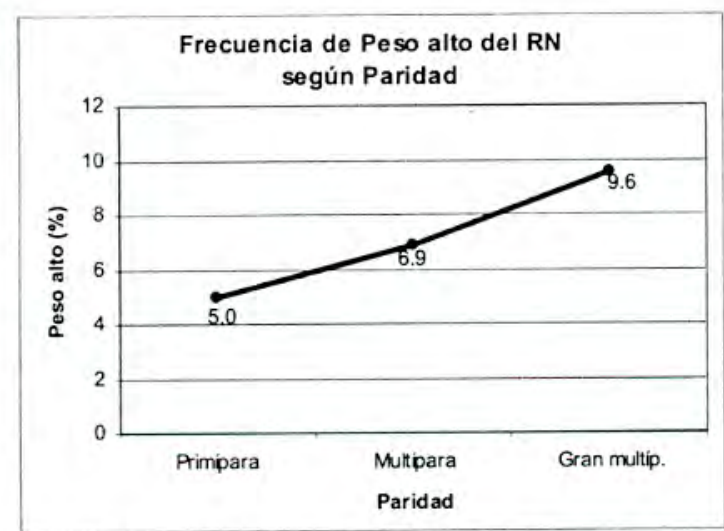

Figura 7. Frecuencia de Peso Alto del RN según paridad Fuente: Sistema Informático Perinatal de Hospitales del MINSAPerú.

\section{DISCUSIÓN}

Durante la gestación ciertas características del ambiente materno ejercen influencia notable sobre el crecimiento intrauterino y su desenlace final el parto, desviándola del curso natural normal genéticamente predeterminado y que pueden implicar un riesgo para la salud del binomio madre-niño.

El peso del recién nacido es un predictor importante de su desarrollo futuro, en particular el bajo peso al nacer (BPN) está asociado con la morbilidad y mortalidad infantil y se ha reportado que es un factor de riesgo importante para la desnutrición proteico-energética en niños menores de 1 año.

Se ha mencionado la influencia de variables independientes, biológicas y sociales, entre otras, para tratar de explicar el bajo peso al nacer; entre los factores de orden biológico se describen edad y talla materna, peso previo al embarazo, paridad, historia reproductiva, nutrición, edad gestacional y enfermedades del embarazo. Dentro de los factores socio económicos se mencionan: ocupación, escolaridad, estado civil y nivel económico.

En este estudio analizaremos la influencia de paridad en el peso del recién nacido. La influencia favorable de la paridad sobre el crecimiento intrauterino podría deberse a mejor perfusión uterina en las multiparas; sin embargo, grados de asociación más significativos se han encontrado en relación con el peso materno; otras variables tienen también efecto aditivo, entre ellas edad, la nutrición y el control de la gestación.

La asociación entre paridad y antropometría del recién nacido muestra que la multiparidad favorece el crecimiento intrauterino. El más alto grado de asociación se observa entre paridad y peso al nacer, asociación estadísticamente significativa. La diferencia del peso promedio al nacer entre los hijos de primíparas y de gran multíparas, es de 230 g.; esta diferencia se relaciona con mejor peso placentario y con aumento de DNA y RNA en la placenta de las multigrávidas, posiblemente un factor asociado al mejor peso del recién nacido en la multípara sea el mayor peso de ésta, otros autores también han encontrado relación entre paridad y peso materno (2).

En nuestro estudio, encontramos un alto porcentaje $(58.2 \%)$ de primiparidad; semejante a lo encontrado por Gonzáles (3) en Huaraz-Perú, $42 \%$ primíparas y $9.7 \%$ grandes multíparas; Herrera (4) en Trujillo- Perú, primíparas $38.9 \%$, multiparas $53.7 \%$ y gran multiparas $7.4 \%$; Banegas (5) en Honduras, encontró bajo porcentaje de primigestas $20.5 \%$ y $11.2 \%$ gran multiparas, Lagos (6) en Temuco-Chile, encontró que la paridad promedio fue 1.26.

El peso promedio de nuestros RN fue $3306 \mathrm{~g} .$, se aprecia un incremento progresivo del peso de los $\mathrm{RN}$ a medida que aumenta la paridad hasta el quinto, a partir del cual disminuye; los RN de madres primíparas tienen $84 \mathrm{~g}$. menos que las gran multíparas. El percentil 50 de peso 
de los RN de madres primiparas fue menor que la población general, mientras que las multíparas y grandes multiparas presentaron pesos mayores. Se asoció bajo peso al nacer y alto peso con gran multiparidad, y peso insuficiente con primíparidad.

Semejantes hallazgos encontraron Ticona (7) en TacnaPerú, en la que los hijos de multiparas superó significativamente en 192 a $238 \mathrm{~g}$. a los de primíparas, de la semana 38 a 42, Juez (8) en Santiago de Chile, encontró 85 a $140 \mathrm{~g}$. de peso neonatal de multiparas mayor que primíparas, entre las semanas 38 a 41 , San Pedro (9) en Buenos Aires-Argentina, $200 \mathrm{~g}$. más de peso en multíparas que en primiparas, Lagos (10) en Temuco-Chile, encontró que las multiparas presentaron RN que pesaron $85 \mathrm{~g}$. más que el promedio (3512 g.) y los RN de primíparas $100 \mathrm{~g}$. menos y Herrera (4) en Trujillo-Perú, encontró incremento de promedios de peso del RN según paridad: primíparas 3090 g., multiparas $3273 \mathrm{~g}$. y gran multiparas $3371 \mathrm{~g}$. $(\mathrm{p}<0.0 \mathrm{l})$. Zhang (11), describe patrones de crecimiento al nacimiento por paridad utilizando certificado de nacimiento de la población de EUA de 1989, en más de 4 millones de RN, demostrando diferencias de peso al nacer en primíparas y multiparas. Lomaglio (12) en Antofagasta de la Sierra - Argentina, encontró paridad promedio de 3.17 hijos; concluye que el peso de nacimiento fue influenciado por la paridad. Jong (13) en Amsterdam -Holanda, Cogswell (14), Mabiala (15) en Brazzaville-Congo, encuentran asociación directa entre paridad y peso al nacer.

Diferentes resultados encontraron otros investigadores como Banegas (5) en Honduras demostró asociación inversa entre paridad y peso al nacer, a mayor paridad mayor bajo peso $(p<0.05)$. El bajo peso al nacer es más frecuente en madres con más de 5 embarazos. Otros autores no encuentran asociación entre el bajo peso con la paridad, como Vázquez (16) en La Habana-Cuba y De Sousa (17) en Piauí-Brasil.

\section{CONCLUSIONES}

Se concluye que la paridad tiene influencia estadisticamente significativa en el peso del RN a término de hospitales del Ministerio de Salud del Perú. Se recomienda tener en cuenta este factor para determinar los grupos de riesgo materno y neonatal.

\section{REFERENCIAS BIBLIOGRÁFICAS}

1. Belitsky R, Benedetti W, De Mucio B, Diaz A. Días J, Estol P, et al. Tecnologias Perinatales. Publicación Cientifica CLAP $N^{\circ}$ 1255. Montevideo, Uruguay. Marzo 1992.

2. Váscones F, Sempértegui F, Naranjo C, Estrella L, Espín V, Arias A, et al. Crecimiento intrauterino en Quito. Universidad Central del Ecuador. Facultad de Ciencias Médicas CONACYT. Quito-Ecuador, 1984.

3. Gonzales G, Tapia V, Serna J. Pajuelo A, Muñoz M, Carrillo C, Peñaranda A. Características de la gestación, del parto y recién nacido en la ciudad de Huaraz, 2001-2005. Acta Med Per. 2006; 23(3):13742.
4. Herrera C, Calderón N, Carvajal R. Influencia de la paridad, edad materna y edad gestacional en el peso del recién nacido. Ginecol Obstet (Perú). 1997;43(2).

5.Banegas O, Pineda E. Influencia de los factores biológicos y socio económicos en el peso de $\mathrm{RN}$ a término, sin malformaciones congénitas aparentes en el Instituto Hondureño de Seguridad Social. Rev Med Post UNAH. 2002; 7(3):285-8.

6. Lagos R, Espinoza R, Orellana J. Antropometría materna y peso promedio de nacimiento. Rev Chil Obstet Ginecol. 2001; 66(2):99-103.

7.Ticona M, Huanco D. Influencia del sexo fetal, paridad y talla materna en el crecimiento intrauterino. Ginecol Obstet (Perú). 2002;48(1):100-4.

8.Juez G, Lucero E, Ventura-Junca P. Crecimiento intrauterino según sexo fetal y paridad materna. Rev Chil Pediatr. 1989; 60(4):204-207.

9. San Pedro M, Grande C, Larguía M, Solana C. Estándar de peso para la edad gestacional en 55706 recién nacidos sanos de una maternidad pública de Buenos Aires. Medicina (Buenos Aires). 2001; $61(1): 15-22$.

10. Lagos R, Espinoza R, Orellana J, Echevarría P. Diferencia en peso de nacimiento promedio según tres variables biológicas en recién nacidos normales. Rev Med Chile. 1999; 127(12).

11. Zhang J, Bowes W. Birth weight for gestational age patterns by race, sex and parity in the United Status population. Obstet Gynecol. 1995; 86(2): 200-8.

12. Lomaglio D, Marrodán $\mathrm{M}$, Verón J, Díaz $\mathrm{M}$, Gallardo F, Alva J, Moreno S. Peso al nacimiento en comunidades de altura en la Puna Argentina: Antofagasta de la Sierra (Catamarca). Antropo. 2005; 9:61-70.

13. Jong C. Atención prenatal óptima aplicando pautas individualizadas. European Journal of Obstetric \& Gynecology and Reproductive Biology (Ed. Española). 2001; 1:95-97.

14. Cogswell M, Yip R. The influence of fetal and maternal factors on the distribution of birthweight. Semin Perinatol. 1995; 19(3):222-40.

15. Mabiala J, Matingou V, Senga P. Risk factors for low birth weight in Brazzaville, Congo. J Gynecol Obstet Biol Reprod (Paris). 2007; 36(8):795-8.

16. Vázquez J, Hernández Y, Farnot U. Caracterización de la madre del recién nacido con bajo peso al nacer. Rev Cubana Obstet Ginecol. 2003;29(2).

17. De Sousa G, Alves de Carvalho H. Influencia de fatores obstétricos, socioeconômicos e nutricionais da gestante sobre o peso do recém-nascido: estudo realizado em uma maternidade em Teresina, Piauí. Rev Brás Saúde Mater Infant. 2004; 4(3).

\section{Correspondencia:}

Manuel Ticona Rendón

Av. Bolognesi $\mathrm{N}^{\circ} 611$ Of. 203. Tacna. Perú manuelticonar@yahoo.es 